\title{
Influence of aging on the peripheral nerve repair process using an artificial nerve conduit
}

\author{
AYAKA KANEKO $^{1}$, KIYOHITO NAITO ${ }^{1}$, SHINJI NAKAMURA ${ }^{2}$, KATSUMI MIYAHARA ${ }^{2}$, KENJI GOTO ${ }^{1}$, \\ HIROYUKI OBATA $^{1}$, NANA NAGURA ${ }^{1}$, YOICHI SUGIYAMA ${ }^{1}$, KAZUO KANEKO $^{1}$ and MUNEAKI ISHIJIMA ${ }^{1}$ \\ ${ }^{1}$ Department of Orthopaedics, Juntendo University School of Medicine; ${ }^{2}$ Laboratory of Morphology and Image Analysis, \\ Research Support Center, Juntendo University Graduate School of Medicine, Bunkyo, Tokyo 113-8421, Japan
}

Received September 14, 2020; Accepted November 24, 2020

DOI: $10.3892 / \mathrm{etm} .2020 .9599$

\begin{abstract}
The influence of aging on the induction of nerve regeneration in artificial nerve conduits has yet to be clarified. In the present study, artificial nerve conduit transplantation and histological analysis using the sciatic nerve of young and elderly mice were performed. Using 20 male C57BL/6 mice, an artificial nerve conduit was transplanted to the sciatic nerve at 8 weeks (Young group) or 70 weeks of age (Aged group), and the sciatic nerve was evaluated histologically at 1, 4 and 12 weeks after surgery. Using hematoxylin and eosin staining, the state of induction of nerve regeneration in the artificial nerve conduit was evaluated. Additionally, immunohistochemical staining was used to investigate an angiogenic marker [vascular endothelial growth factor A (VEGFA)], Schwann cell markers [sex determining region Y-box 10 (SOX10) and S100 calcium-binding protein $\beta$ (S100 $\beta$ )] and a nerve damage marker [nerve growth factor (NGF)]. The results revealed that the induction of nerve regeneration was significantly higher in the Young group than in the Aged group. In addition, VEGFA and SOX10 expression at 1 week, SOX10 expression at 4 weeks and SOX10, S100 $\beta$ and NGF expression at 12 weeks in the proximal stump were significantly higher in the Young group than in the Aged group. At the center of the artificial nerve conduit, $S 100 \beta$ and NGF expression at 4 weeks, and VEGFA, SOX10, S100 $\beta$ and NGF expression at 12 weeks were significantly higher in the Young group than in the Aged group. In the distal stump, no significant difference was noted in immunostaining at any week between the two groups. The present study suggested that the nerve regeneration-inducing functions decrease due to aging.
\end{abstract}

Correspondence to: Dr Kiyohito Naito, Department of Orthopaedics, Juntendo University School of Medicine, 2-1-1 Hongo, Bunkyo, Tokyo 113-8421, Japan

E-mail: knaito@juntendo.ac.jp

Key words: aging, artificial nerve conduits, nerve growth factor, peripheral nerve, Schwann cell, vascular endothelial growth factor

\section{Introduction}

Artificial nerve conduits have recently become used for peripheral nerve damage with a long nerve defect and damage of multiple peripheral nerves in which the nerve stumps cannot be directly sutured (1-3). However, there are several problems with artificial nerve conduits. Decreased angiogenesis, a decrease in nerve fibers, and reduction of axon regeneration were previously reported in artificial nerve conduits when an artificial nerve conduit was used for a nerve defect that was long to a certain extent (4).

On the other hand, Biasibetti et al reported that decreased angiogenesis, a decrease in nerve fibers, and myelin degeneration are age-associated changes in peripheral nerves (5). Furthermore, we previously reported a mechanism to prevent oxidative stress-induced peripheral nerve degeneration (6). Peripheral nerves are functionally and morphologically impaired due to aging (5-7).

Based on the above, the use of artificial nerve conduits is expected to be limited for the elderly whose peripheral nerves are considered to have low axon regenerative ability. However, the influence of aging on the induction of nerve regeneration in artificial nerve conduits has yet to be clarified. Thus, in this study, to histologically evaluate the influence of aging on the induction of peripheral nerve regeneration in artificial nerve conduits, we performed artificial nerve conduit transplantation to the sciatic nerve in young and elderly mice. In addition, histological changes after artificial nerve conduit transplantation in each mouse were compared using hematoxylin and eosin (H-E) and immunohistochemical staining.

\section{Materials and methods}

Animal model. The present study was approved by the Animal Care Committee of Juntendo University, Tokyo, Japan (registration no. 1398; approval no. 310212).

Thirty male C57BL/6 mice purchased from Japan SLC, Inc. were used. To histologically evaluate the influence of aging on the induction of peripheral nerve regeneration in artificial nerve conduits, an artificial nerve conduit was surgically transplanted to the sciatic nerve at 8 (Young group, $n=15$ ) or 70 weeks of age (Aged group, $n=15$ ), and the sciatic nerve was collected and histologically evaluated at 1,4 , and 12 weeks 
after surgery. The mice were maintained at 5 per cage in a sterile environment, and had free access to water and 15-kGy gamma-irradiated feed, CRF-1 (Oriental Yeast Co., Ltd.), at a room temperature of $22 \pm 2^{\circ} \mathrm{C}$ and $40-60 \%$ humidity under a 12-h light and 12-h dark environment.

Preparation of the nerve conduit. The collagen artificial nerve conduit was prepared according to the Nipro patent (United States patent US 6953482 B2). Briefly, enzyme-solubilized collagen (a mixture of collagen types I and III; Nippon Ham, Ibaragi, Japan) was dissolved in water to prepare a 5\% aqueous solution and extruded in a coagulating liquid to produce a collagen fiber with a diameter of $50 \mathrm{~nm}$. The collagen fiber was wound around a metal mandrel to produce a tubular structure consisting of collagen with an inner diameter of $1 \mathrm{~mm}$, wall thickness of $0.25 \mathrm{~mm}$, and length of $50 \mathrm{~mm}$. The tube was filled with 60 collagen filaments (50 nm in diameter) aligned longitudinally with the $5 \%$ aqueous collagen solution. The constructs were rapidly frozen and then lyophilized in vacuo. The construct contained $10 \% \mathrm{v} / \mathrm{v}$ collagen filaments under dry conditions. The collagen artificial nerve conduit was then subjected to 25-kGy gamma-ray irradiation for sterilization.

Surgical procedures. Under generalized anesthesia with isoflurane inhalation anesthetic solution (4\% isoflurane used for induction and $2 \%$ for maintenance), a skin incision was made on the lateral side of the right hind limb. For manipulation of the sciatic nerve, a light microscope (Zeiss Axioskop2; magnification, $\mathrm{x} 40$ ) was used. The sciatic nerve was dissected from the surrounding tissue (Fig. 1A) and cut to prepare a 3-mm defect. The nerve defect was bridged by the collagen artificial nerve conduit (Fig. 1B). The proximal and distal nerve stumps were both placed within the conduit, and epineural sutures were made using 9-0 nylon, as described previously by Fields et al (Fig. 1C) (8).

The postoperative activity of the mice was not limited and they were maintained the same as in the preoperative environment. Under generalized anesthesia with isoflurane inhalation anesthetic solution (4\% isoflurane used for induction and $2 \%$ for maintenance), the right sciatic nerves including collagen nerve conduits from both groups were harvested at 1, 4, and 12 weeks after surgery. Mice were sacrificed by cervical dislocation on the day the sciatic nerves were harvesting.

$H$-E staining assessment of the age-associated change in the peripheral nerve. The collected sciatic nerve was fixed in $4 \%$ paraformaldehyde at room temperature for $72 \mathrm{~h}$ and paraffin blocks were prepared. Tissue sections were prepared by cutting the sciatic nerve in the minor axis direction at $3-\mu \mathrm{m}$ thickness in the following positions: (Proximal): The sciatic nerve proximal to the artificial nerve conduit; (Center): The central region of the artificial nerve conduit; and (Distal): The sciatic nerve distal to the artificial nerve conduit (Fig. 2). H-E staining was performed to evaluate the state of absorption of the outer cylinder of the artificial nerve conduit at Center and state of nerve regeneration induction in the artificial nerve conduit. Using a light microscope (Olympus, AX73), the cross-sectional area including the outer cylinder of the artificial nerve conduit (I), that of the inner lumen of the artificial nerve conduit in the region filled with nerve fibers (II),
A

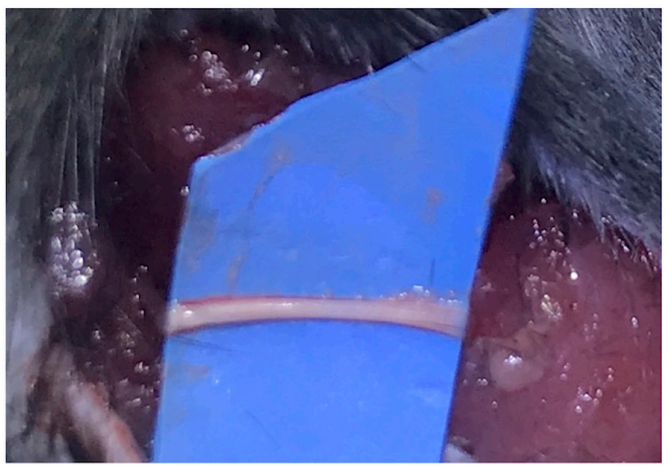

B

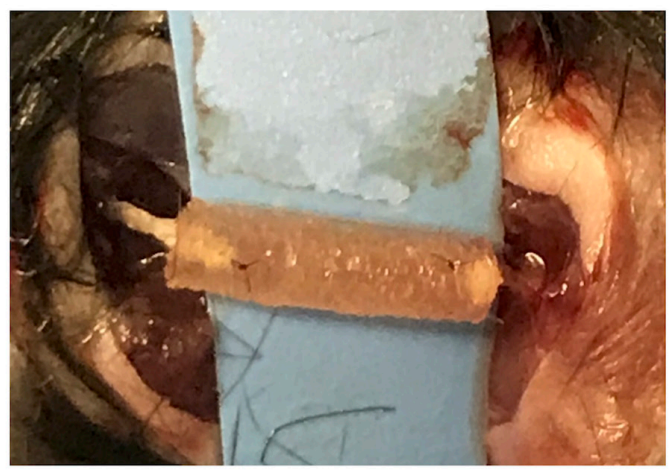

C

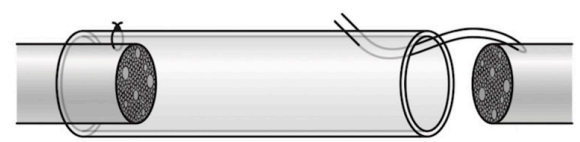

Figure 1. Nerve repair method using artificial nerve conduits. (A) The sciatic nerve was dissected from the surrounding tissue. (B) The mouse right sciatic nerve was cut, and a 3-mm defect was prepared. The nerve defect was bridged by the collagen artificial nerve conduit. (C) The proximal and distal nerve stumps were both placed within the conduit, and epineural sutures were made using 9-0 nylon. Scale bar, $1 \mathrm{~mm}$.

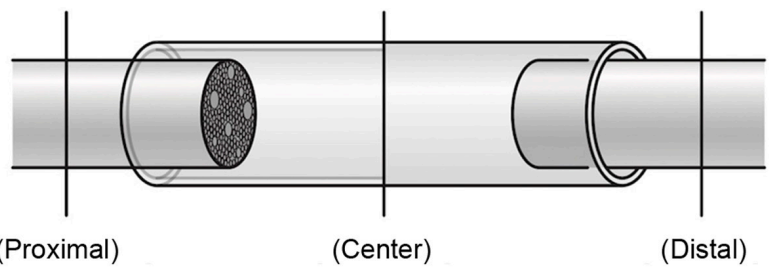

Figure 2. Positions of tissue sections. Tissue sections were prepared by cutting the sciatic nerve at $3-\mu \mathrm{m}$ thickness in the minor axis direction at the following positions: Proximal, the sciatic nerve proximal to the artificial nerve conduit; Center, central region of the artificial nerve conduit; and Distal, the sciatic nerve distal to the artificial nerve conduit.

and the rate of the inner lumen of the artificial nerve conduit being filled with nerve fibers (III=II/I x100) were calculated to evaluate the state of induction of nerve regeneration in the artificial nerve conduit.

Histochemical assessment of the age-related change in the peripheral nerve. Several peripheral nerve-specific proteins were immunohistochemically stained. The antibodies used were those against vascular endothelial growth factor A (VEGFA) as an angiogenic marker, sex-determining region Y-box 10 (SOX10) and S100 calcium-binding protein $\beta$ (S100 $\beta)$ 
as Schwann cell markers, and nerve growth factor (NGF) as a marker for nerve damage. The primary antibodies and secondary biotinylated antibodies are summarized in Table I.

After deparaffinization, antigen activation treatment for SOX10 and S100 $\beta$ was performed at $121^{\circ} \mathrm{C}$ for $10 \mathrm{~min}$ using an autoclave, whereas VEGFA and NGF did not require antigen activation treatment. Then, to block endogenous biotin, the sections were treated with the Avidin/Biotin Blocking Kit (Vector ${ }^{\mathrm{R}}$, SP-2001) and blocked with $2 \%$ bovine serum albumin (BSA) (Sigma-Aldrich, A2153) in PBS for $30 \mathrm{~min}$ at room temperature, followed by reactions with primary antibodies against the different proteins at $4^{\circ} \mathrm{C}$ for $15 \mathrm{~h}$. After treatment with the primary antibody, the sections were immersed in $3 \%$ hydrogen peroxide for 5 min to block endogenous peroxidase. Subsequently, the sections were reacted with the secondary antibodies against the proteins at room temperature for $40 \mathrm{~min}$. Lastly, the sections were reacted with peroxidase-conjugated streptavidin (Dako, P397) at room temperature for $40 \mathrm{~min}$. The level of protein expression in the nerve was quantitated in each section using the Nuance FX (PerkinElmer) multispectral camera system. The area stained in brown was regarded as positive, and the ratio of the area of the positive section to the area of the short-axis section of the sciatic nerve was calculated.

Statistical analysis. Data are presented as the mean \pm standard deviation (SD) and were analyzed for significant differences by the Mann-Whitney U test (Prism 4; GraphPad Software). Differences were considered significant at $\mathrm{P}<0.05$.

\section{Results}

The cross-sectional area (I) including the outer cylinder of the artificial nerve conduit on $\mathrm{H} \& \mathrm{E}$ staining was $2.19 \pm 0.11 \mathrm{~mm}^{2}$ in the Young group and $2.18 \pm 0.15 \mathrm{~mm}^{2}$ in the Aged group at 1 week $(P>0.99), 1.28 \pm 0.21 \mathrm{~mm}^{2}$ in the Young group and $1.75 \pm 0.36 \mathrm{~mm}^{2}$ in the Aged group at 4 weeks $(\mathrm{P}=0.06)$, and $0.22 \pm 0.06 \mathrm{~mm}^{2}$ in the Young group and $0.56 \pm 0.31 \mathrm{~mm}^{2}$ in the Aged group at 12 weeks $(\mathrm{P}=0.11)$. The cross-sectional area (II) of the inner lumen of the artificial nerve conduit filled with nerve fibers was $0.26 \pm 0.02 \mathrm{~mm}^{2}$ in the Young group and $0.24 \pm 0.05 \mathrm{~mm}^{2}$ in the Aged group at 1 week $(\mathrm{P}=0.55)$, $0.26 \pm 0.06 \mathrm{~mm}^{2}$ in the Young group and $0.20 \pm 0.05 \mathrm{~mm}^{2}$ in the Aged group at 4 weeks $(\mathrm{P}=0.22)$, and $0.15 \pm 0.04 \mathrm{~mm}^{2}$ in the Young group and $0.11 \pm 0.02 \mathrm{~mm}^{2}$ in the Aged group at 12 weeks $(\mathrm{P}=0.19)$. The rate of the inner lumen of the artificial nerve conduit being filled with nerve fibers (III) was $11.7 \pm 0.8 \%$ in the Young group and $11.1 \pm 2.5 \%$ in the Aged group at 1 week $(\mathrm{P}=0.42), 21.4 \pm 7.6 \%$ in the Young group and $12.1 \pm 4.5 \%$ in the Aged group at 4 weeks $(P<0.05)$, and $67.2 \pm 5.5 \%$ in the Young group and $26.4 \pm 12.8 \%$ in the Aged group at 12 weeks $(\mathrm{P}<0.05)$. (III) was significantly higher in the Young group than in the Aged group at 4 and 12 weeks (Fig. 3).

Regarding the expression of each protein in the peripheral nerve, the anti-VEGFA antibody-positive expression rates at Proximal in the Young and Aged groups were $17.4 \pm 3.3$ and $3.1 \pm 2.3 \%$, respectively, at 1 week $(\mathrm{P}<0.05)$, $8.4 \pm 2.7$ and $7.8 \pm 2.7 \%$, respectively, at 4 weeks $(\mathrm{P}=0.69)$, and $6.2 \pm 2.1$ and $3.6 \pm 1.2 \%$, respectively, at 12 weeks $(\mathrm{P}=0.06)$. At Center, the rates were $0.0 \pm 0.0$ and $0.0 \pm 0.0 \%$, respectively,

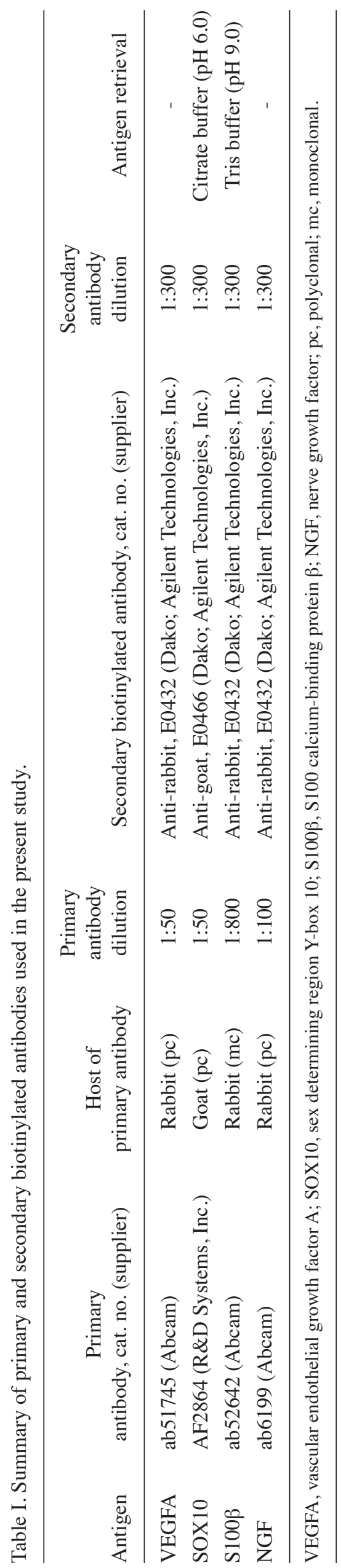



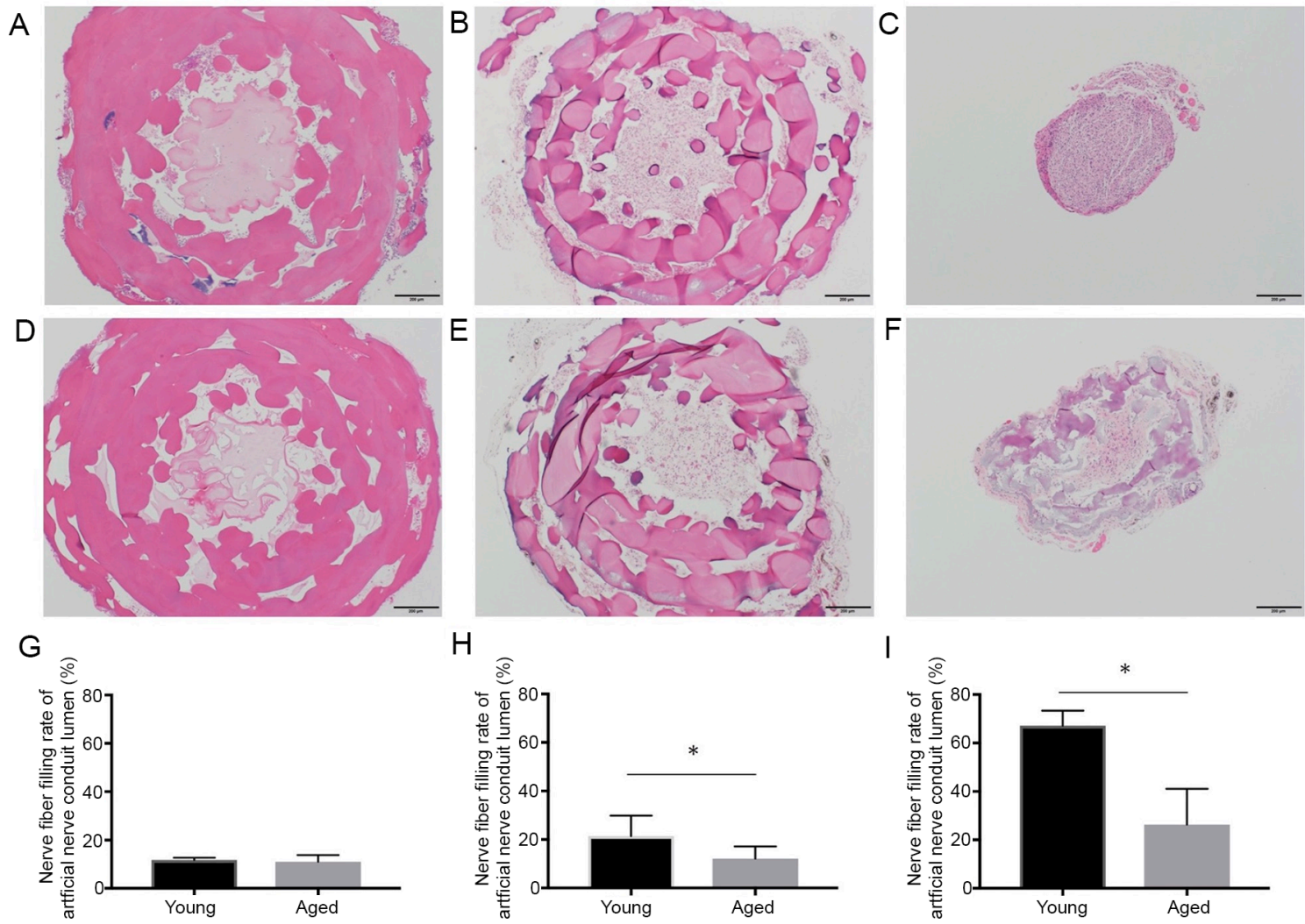

Figure 3. Histological analysis by hematoxylin and eosin staining. Young group at postoperative (A) 1 week, (B) 4 weeks and (C) 12 weeks (Center). Aged group at postoperative (D) 1 week, (E) 4 weeks and (F) 12 weeks (Center). Scale bar, $200 \mu \mathrm{m}$. Rate of the inner lumen of the artificial nerve conduit being filled with nerve fibers at postoperative (G) 1 week, (H) 4 weeks and (I) 12 weeks. ${ }^{*} \mathrm{P}<0.05$.

at 1 week $(\mathrm{P}>0.99), 4.7 \pm 2.2$ and $2.4 \pm 1.7 \%$, respectively, at 4 weeks $(\mathrm{P}=0.10)$, and $4.2 \pm 0.4$ and $2.9 \pm 0.8 \%$, respectively, at 12 weeks $(\mathrm{P}<0.05)$. At Distal, the rates were $5.0 \pm 2.2$ and $4.5 \pm 2.5 \%$, respectively, at 1 week $(\mathrm{P}=0.69), 4.7 \pm 1.7$ and $4.2 \pm 2.8 \%$, respectively, at 4 weeks $(\mathrm{P}>0.99)$, and $3.9 \pm 1.3$ and $2.8 \pm 1.3 \%$, respectively, at 12 weeks $(\mathrm{P}=0.25)$. The VEGFA expression rate at Proximal was significantly higher in the Young group than in the Aged group at postoperative 1 week, and the rate at Center was significantly higher in the Young group than in the Aged group at postoperative 12 weeks, whereas no significant difference was noted in the rate at Distal between the 2 groups at any week (Fig. 4).

The anti-SOX10 antibody-positive expression rates at Proximal in the Young and Aged groups were $1.6 \pm 0.6$ and $0.6 \pm 0.2 \%$, respectively, at 1 week $(P<0.05)$, $3.4 \pm 0.4$ and $1.7 \pm 0.3 \%$, respectively, at 4 weeks $(\mathrm{P}<0.05)$, and $3.1 \pm 0.9$ and $2.2 \pm 0.3 \%$, respectively, at 12 weeks $(\mathrm{P}<0.05)$. The rates at Center were $0.0 \pm 0.0$ and $0.0 \pm 0.0 \%$, respectively, at 1 week $(\mathrm{P}>0.99), 2.1 \pm 1.7$ and $0.8 \pm 0.4 \%$, respectively, at 4 weeks $(\mathrm{P}=0.11)$, and $4.7 \pm 1.1$ and $1.5 \pm 0.6 \%$, respectively, at 12 weeks $(\mathrm{P}<0.05)$. The rates at Distal were $1.6 \pm 0.2$ and $1.5 \pm 0.4 \%$, respectively, at 1 week $(\mathrm{P}=0.65), 2.3 \pm 0.5$ and $1.8 \pm 0.6 \%$, respectively, at 4 weeks $(\mathrm{P}=0.41)$, and $2.9 \pm 1.6$ and $2.3 \pm 0.6 \%$, respectively, at 12 weeks $(\mathrm{P}>0.99)$. The SOX10 expression rate at Proximal was significantly higher in the Young group than in the Aged group at postoperative 1,4 , and 12 weeks, and the rate at Center was significantly higher in the Young group than in the Aged group at postoperative 12 weeks. No significant difference was noted in the SOX10 expression rate at Distal between the 2 groups at any week (Fig. 5).
The S100 $\beta$ expression rates at Proximal in the Young and Aged groups were $7.5 \pm 1.6$ and $5.4 \pm 1.5 \%$, respectively, at 1 week $(\mathrm{P}=0.15), 10.0 \pm 1.9$ and $7.0 \pm 1.2 \%$, respectively, at 4 weeks $(\mathrm{P}=0.06)$, and $14.2 \pm 2.5$ and $7.0 \pm 1.6 \%$, respectively, at 12 weeks $(\mathrm{P}<0.05)$. The rates at Center were $0.0 \pm 0.0$ and $0.0 \pm 0.0 \%$, respectively, at 1 week $(\mathrm{P}>0.99), 1.5 \pm 0.9$ and $0.6 \pm 0.1 \%$, respectively, at 4 weeks $(\mathrm{P}<0.05)$, and $3.9 \pm 1.2$ and $1.4 \pm 0.2 \%$, respectively, at 12 weeks $(\mathrm{P}<0.05)$. The rates at Distal were $5.0 \pm 0.6$ and $4.4 \pm 1.2 \%$, respectively, at 1 week $(\mathrm{P}=0.41)$, $5.6 \pm 0.7$ and $4.8 \pm 0.4 \%$, respectively, at 4 weeks $(\mathrm{P}=0.19)$, and $6.7 \pm 0.7$ and $5.1 \pm 0.9 \%$, respectively, at 12 weeks $(\mathrm{P}=0.19)$. The S100 $\beta$ expression rate at Proximal was significantly higher in the Young group than in the Aged group at postoperative 12 weeks. The S100 $\beta$ expression rate at Center was significantly higher in the Young group than in the Aged group at postoperative 4 and 12 weeks. No significant difference was noted in the $\mathrm{S} 100 \beta$ expression rate at Distal between the 2 groups at any week (Fig. 6).

The NGF expression rates at Proximal in the Young and Aged groups were $7.0 \pm 2.5$ and $5.1 \pm 0.9 \%$, respectively, at 1 week $(\mathrm{P}=0.22), 8.9 \pm 1.5$ and $6.8 \pm 1.6 \%$, respectively, at 4 weeks $(\mathrm{P}=0.25)$, and $7.0 \pm 1.2$ and $4.5 \pm 1.6 \%$, respectively, at 12 weeks $(\mathrm{P}<0.05)$. The rates at Center were $0.0 \pm 0.0$ and $0.0 \pm 0.0 \%$, respectively, at 1 week $(\mathrm{P}>0.99), 7.0 \pm 2.0$ and $2.6 \pm 0.7 \%$, respectively, at 4 weeks $(\mathrm{P}<0.05)$, and $6.5 \pm 0.9$ and $3.4 \pm 1.0 \%$, respectively, at 12 weeks $(\mathrm{P}<0.05)$. The rates at Distal were $5.4 \pm 1.4$ and $4.4 \pm 1.3 \%$, respectively, at 1 week $(\mathrm{P}=0.42), 7.5 \pm 2.4$ and $5.8 \pm 0.9 \%$, respectively, at 4 weeks $(\mathrm{P}=0.29)$, and $5.5 \pm 0.7$ and $4.4 \pm 1.6 \%$, respectively, at 12 weeks $(\mathrm{P}=0.39)$. The NGF expression rate at Proximal was 


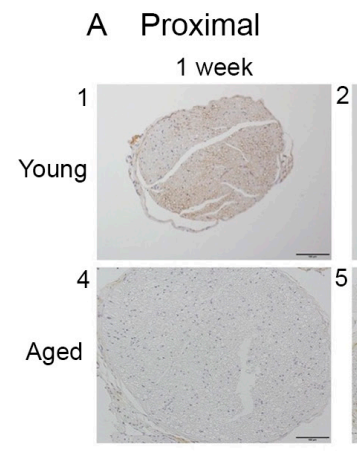

B Center

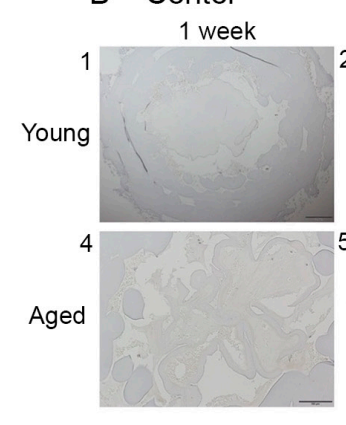

C Distal

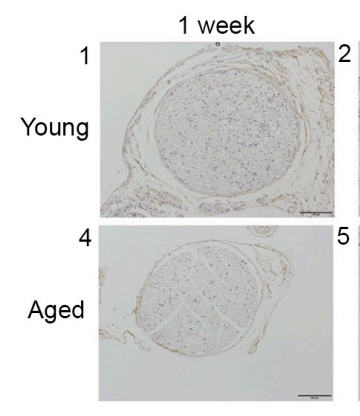

4 week

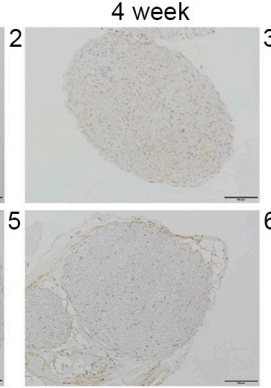

4 week

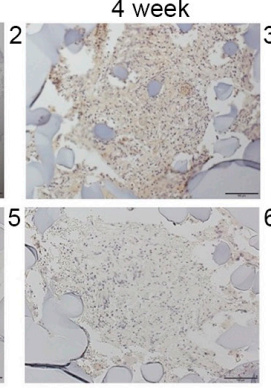

4 week

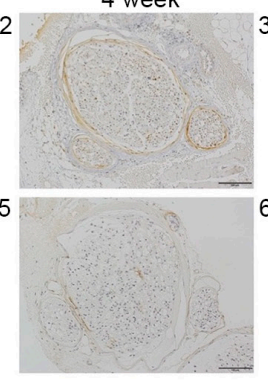

12 week

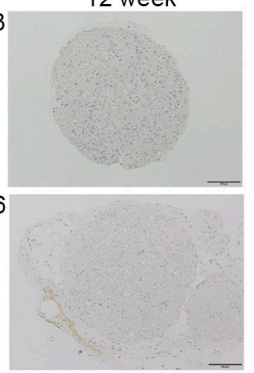

12 week

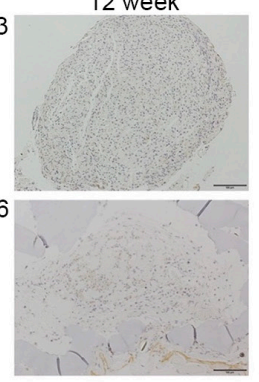

12 week

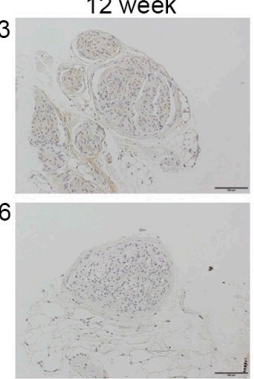

D Proximal

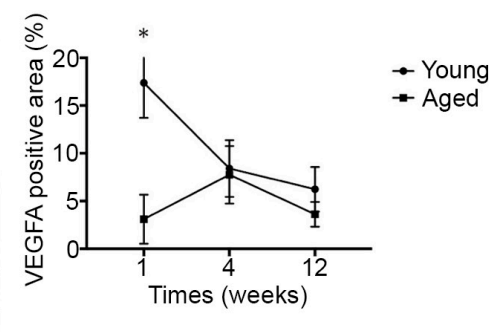

E Center

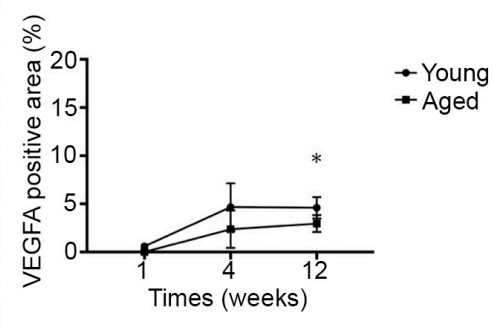

F Distal

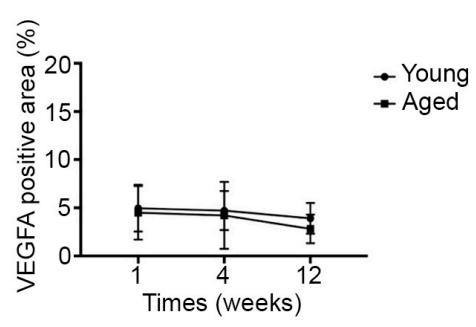

Figure 4. VEGFA expression in the regenerated peripheral nerve. (A) Images of VEGFA immunohistochemical staining at the Proximal position. (B) VEGFA expression rate at the Proximal position. (C) Images of VEGFA immunohistochemical staining at the Center position. (D) VEGFA expression rate at the Center position. (E) Images of VEGFA immunohistochemical staining at the Distal position. (F) VEGFA expression rate at the Distal position. Scale bar, $100 \mu \mathrm{m}$. ${ }^{*} \mathrm{P}<0.05$ vs. Aged group. VEGFA, vascular endothelial growth factor A.

significantly higher in the Young group than in the Aged group at postoperative 12 weeks. The NGF expression rate at Center was significantly higher in the Young group than in the Aged group at postoperative 4 and 12 weeks. No significant difference was noted in the NGF expression rate at Distal between the 2 groups at any week (Fig. 7).

\section{Discussion}

Several previous studies reported that the peripheral nerve regeneration ability decreases due to aging $(9,10)$. In this study, the rate of the inner lumen of the artificial nerve conduit being filled with nerve fibers (III) was significantly lower in the Aged group than in the Young group at 4 and 12 weeks after artificial nerve conduit transplantation, suggesting aging-induced reduction of peripheral nerve regeneration ability. 'Why is nerve regenerative ability reduced by aging?' Verdú et al reported that Schwann cell function declines due to aging, delaying Waller degeneration and axon regeneration after peripheral nerve damage (10). In addition, the vascular regeneration ability of nerve tissue after peripheral nerve damage was recently demonstrated to decrease with age $(5,11)$. On the other hand, the generation of new blood vessels starting from the proximal nerve stump after peripheral nerved damage is important $(12,13)$. Furthermore, Schwann cell migration along the new blood vessel generated in this manner was clarified to promote axon regeneration (12). Thus, we performed immunostaining to elucidate the difference in the rate of the artificial nerve conduit inner lumen being filled with nerve fibers (III) between the Young and Aged groups on H\&E staining.

VEGFA promotes new blood vessel generation, playing an important role in the early step of nerve regeneration, and it is expressed a few days after peripheral nerve damage $(12,13)$. In the present study, VEGFA expression at Proximal was significantly lower in the Aged group than that in the Young group at postoperative 1 week, and VEGFA expression in the Aged group did not exceed that in the Young group at postoperative 4 or 12 weeks, confirming that VEGFA expression ability decreases due to aging. At Center, VEGFA was gradually 
A Proximal

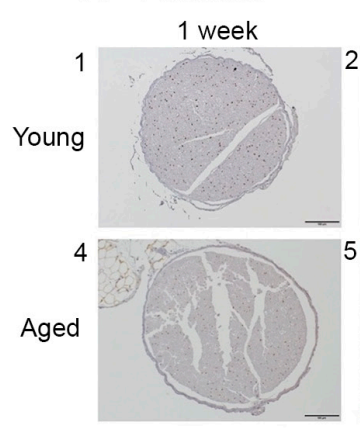

B Center

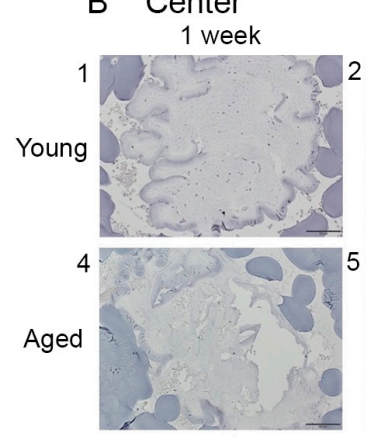

C Distal

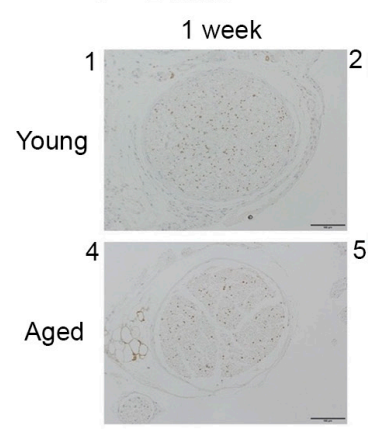

4 week

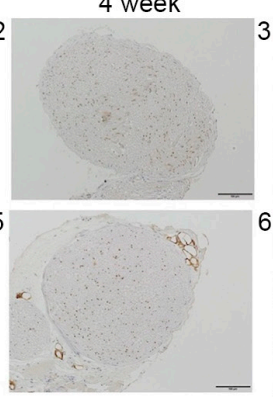

4 week

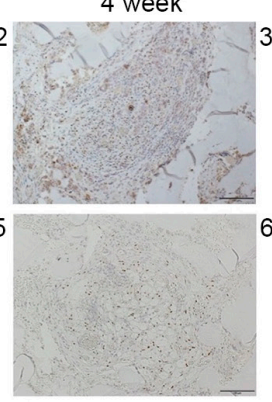

4 week

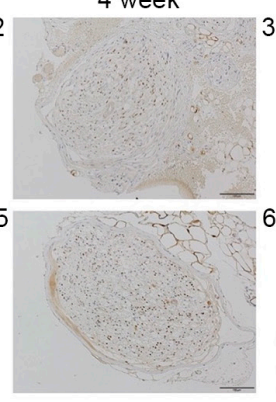

12 week

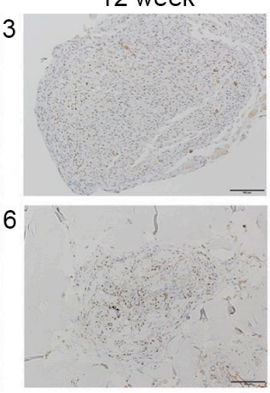

12 week

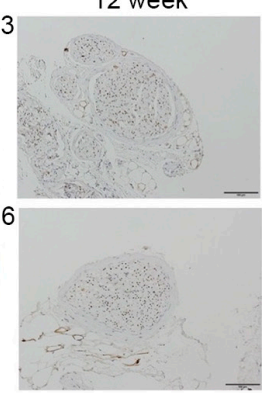

D Proximal

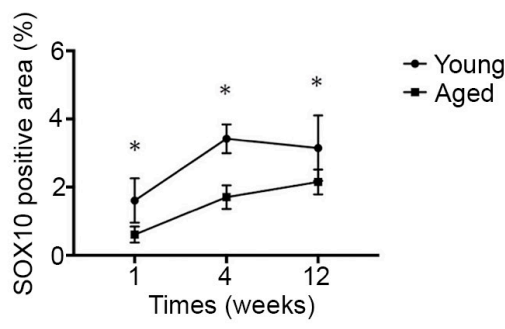

E Center

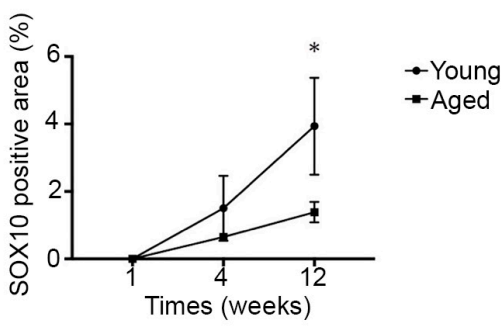

F Distal

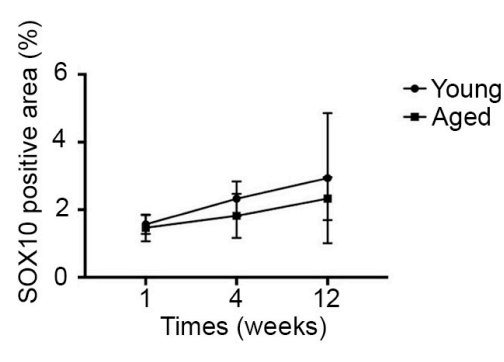

Figure 5. SOX10 expression in the regenerated peripheral nerve. (A) Images of SOX10 immunohistochemical staining at the Proximal position. (B) SOX10 expression rate at the Proximal position. (C) Images of SOX10 immunohistochemical staining at the Center position. (D) SOX10 expression rate at the Center position. (E) Images of SOX10 immunohistochemical staining at the Distal position. (F) SOX10 expression rate at the Distal position. Scale bar, $100 \mu \mathrm{m}$. ${ }^{*} \mathrm{P}<0.05$ vs. Aged group. SOX10, sex determining region Y-box 10.

expressed from postoperative 1 week to 12 weeks in both groups, and new blood vessels were observed at postoperative 4 weeks on H\&E staining, suggesting that VEGFA induced new blood vessel generation from the proximal nerve stump toward the artificial nerve conduit. Generation of these new blood vessels exhibited the same pattern as that reported by Cattin et al (12). Based on the above, aging-induced reduction of VEGFA expression early after peripheral nerve damage was suggested to delay nerve regeneration.

Regarding the appearance of Schwann cells migrating along the new blood vessel, immunohistological staining was performed focusing on SOX10 expressed at all steps of Schwann cell development from the early step of neural crest cells to S100 $\beta$ expression by differentiated Schwann cells (14-16). As SOX10 expression at Proximal increased at postoperative 1, 4, and 12 weeks in the Young group, Schwann cells may have appeared with VEGFA at Proximal in the early step of nerve regeneration in the Young group. The increase in $\mathrm{S} 100 \beta$ at Proximal at postoperative 12 weeks in the Young group may have been induced by SOX10, which is consistent with the finding reported by Fujiwara et al that 'induction of $\mathrm{S} 100 \beta$ expression is one of the important functions of SOX10' (14), i.e., Schwann cell maturation with migration along the new blood vessel at Proximal was observed in the Young group. On the other hand, in the Aged group, SOX10 expression at Proximal was lower at postoperative 1, 4, and 12 weeks than that in the Young group, confirming that Schwann cell migration is not readily induced at the proximal nerve stump due to aging. At Center, S100 $\beta$ expression increased at postoperative 4 weeks in the Young group. This reaction at Center in the Young group may have been due to increases in VEGFA and SOX10 expression at Proximal, which induced Schwann cell migration along the new blood vessel from Proximal to Center, promoting Schwann cell differentiation. Furthermore, S100 $\beta$ expression at Center in the Aged group was significantly lower than that in the Young group at postoperative 4 and 12 weeks. Although evaluation of aging-associated Schwann cell differentiation ability is difficult, this study revealed that Schwann cell migration ability markedly 
A Proximal

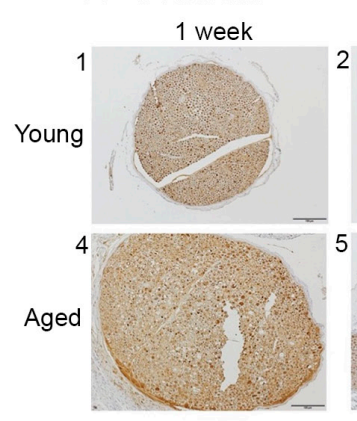

B Center

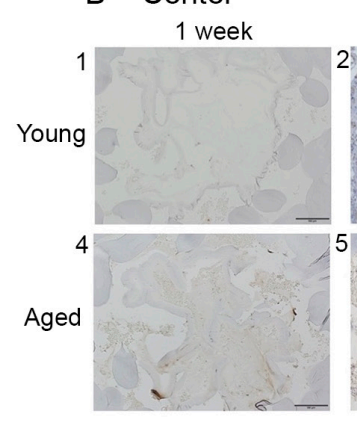

C Distal

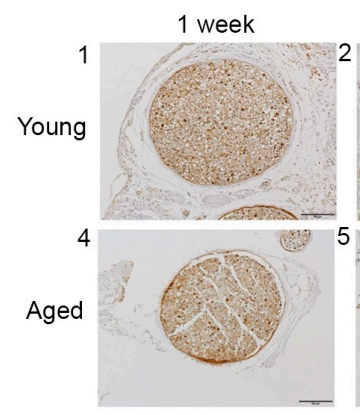

4 week

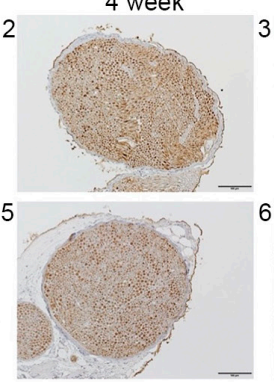

6

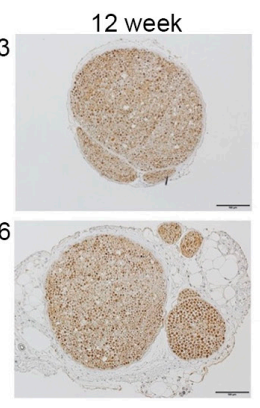

12 week
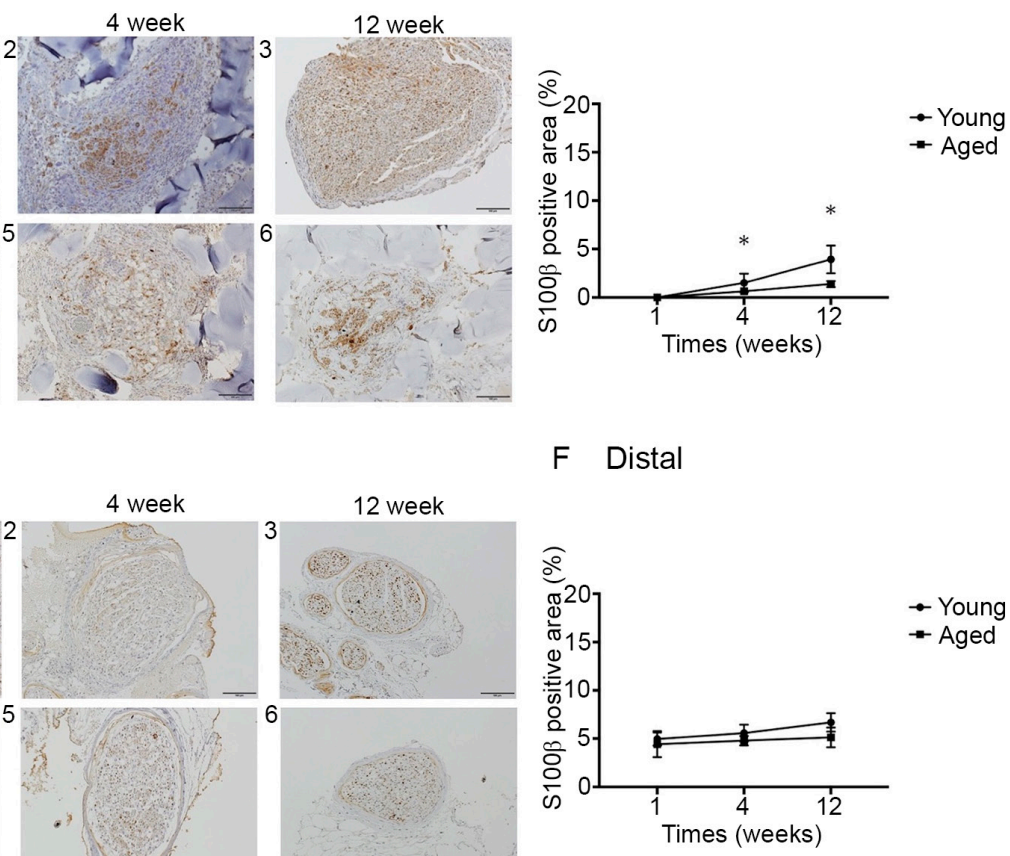

F Distal

$\rightarrow$ Young

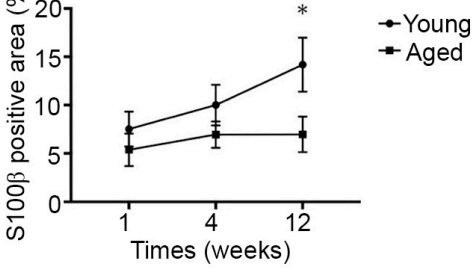

E Center

$\rightarrow$ Young

- Aged

Figure 6. S100 $\beta$ expression in the regenerated peripheral nerve. (A) Images of S100 $\beta$ immunohistochemical staining at the Proximal position. (B) S100 $\beta$ expression rate at the Proximal position. (C) Images of S100 $\beta$ immunohistochemical staining at the Center position. (D) S100 $\beta$ expression rate at the Center position. (E) Images of S100 $\beta$ immunohistochemical staining at the Distal position. (F) S100 $\beta$ expression rate at the Distal position. Scale bar, $100 \mu \mathrm{m}$. ${ }^{*} \mathrm{P}<0.05$ vs. Aged group. $\mathrm{S} 100 \beta, \mathrm{S} 100$ calcium-binding protein $\beta$.

decreased due to aging and mature Schwann cell development also decreased, suggesting that Waller degeneration during in the nerve regeneration process and axon regeneration ability are reduced by aging.

NGF, a marker of nerve damage, has neuroprotective and nerve/axon regenerative functions, which decline with age (17-21). NGF is present at a low level in normal nerve tissue, but upon nerve damage, NGF production is promoted by Schwann cells (22-24). Based on this study, NGF expression at Proximal at postoperative 12 weeks, and Center at postoperative 4 and 12 weeks was significantly higher in the Young group. Such NGF expression was similar to that of $\mathrm{S} 100 \beta$ expression, representing mature Schwann cell development, suggesting that NGF was expressed by mature Schwann cells, i.e., mature Schwann cell development decreases in peripheral nerves damaged by aging, reducing the expression of NGF with nerve/axon regenerative function and delaying peripheral nerve regeneration.

In damaged peripheral nerves, Schwann cell migration along the new blood vessel starting from the proximal nerve stump promotes axon regeneration. In the peripheral nerve after artificial nerve conduit transplantation in the Aged group, VEGFA (new blood vessel), SOX10 and S100ß (Schwann cells), and NGF (axon regeneration) expression representing the axon regeneration process was significantly lower than that in the Young group, suggesting that peripheral nerve regeneration is delayed by aging. As no study of the efficacy and safety of artificial nerve conduits in the elderly has been performed in Japan, their use for elderly patients has to be carefully investigated. In addition, based on this study, the efficacy of the use of an artificial nerve conduit in elderly patients is inferior to that in young patients. Thus, surgical treatment using conventional artificial nerve conduits for nerve injuries in the elderly has limitations; therefore, new additional therapies aimed at axonal regeneration need to be developed for elderly patients. Detailed elucidation of age-associated changes in peripheral nerves in the future may help to prevent and treat peripheral nerve disorder in an aged society.

There is a limitation of this study which is the lack of investigation of the motor function of the lower limbs. The mice in both Young and Aged group had motor paralysis with 


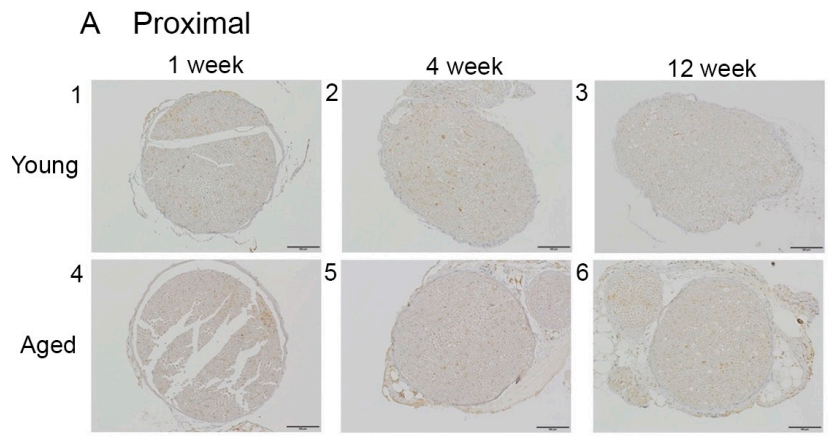

D Proximal
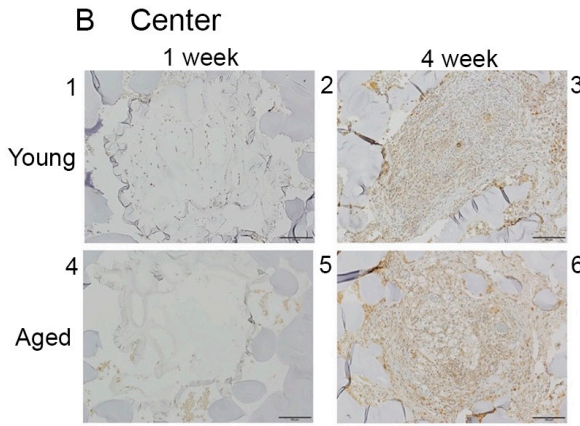

12 week

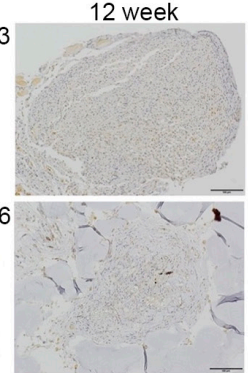

E Center

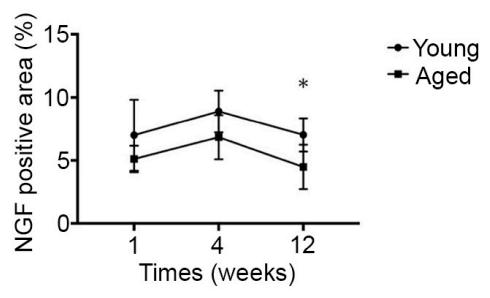

C Distal

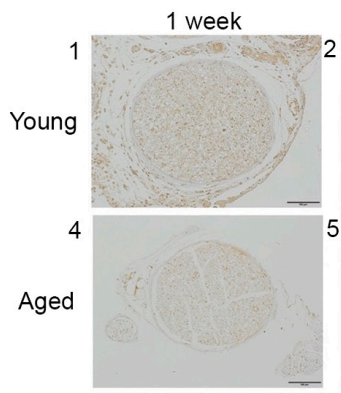

4 week

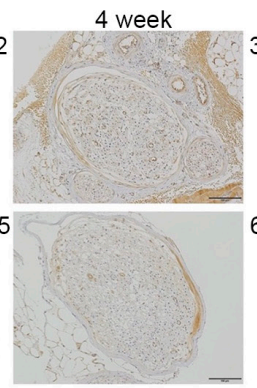

12 week

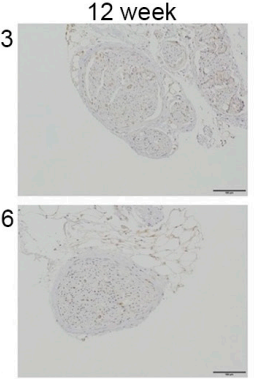

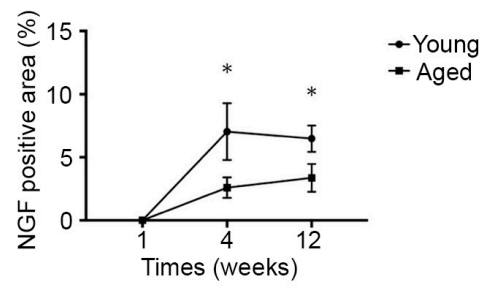

F Distal

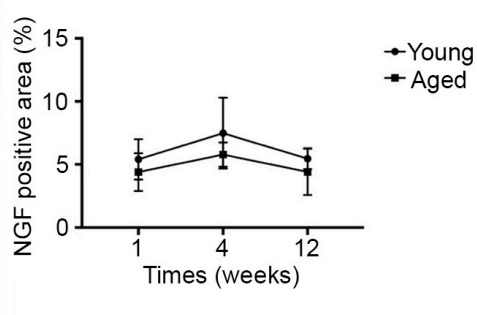

Figure 7. NGF expression in the regenerated peripheral nerve. (A) Images of NGF immunohistochemical staining at the Proximal position. (B) NGF expression rate at the Proximal position. (C) Images of NGF immunohistochemical staining at the Center position. (D) NGF expression rate at the Center position. (E) Images of NGF immunohistochemical staining at the Distal position. (F) NGF expression rate at the Distal position. Scale bar, $100 \mu \mathrm{m}$. ${ }^{*}<0.05$ vs. Aged group. NGF, nerve growth factor.

limping after surgery. However, we had focused on histopathological evaluation of the peripheral nerve and not investigated the motor function of the lower limbs.

In damaged peripheral nerves, axon regeneration is promoted by Schwann cell migration along a new blood vessel starting from the proximal nerve stump. This study suggested that these nerve regeneration-inducing functions decrease with age in the nerve regeneration induction process in artificial nerve conduits.

\section{Acknowledgements}

Not applicable.

\section{Funding}

The present study was supported by Nipro Corporation.

\section{Availability of data and materials}

The datasets used and/or analyzed during the current study are available from the corresponding author on reasonable request.

\section{Authors' contributions}

AK mainly wrote the manuscript and acquired, analyzed and interpretated the data. KN wrote the manuscript and made substantial contributions to conception and design of the study, and interpretation of data. SN, KM and YS contributed to acquisition, analysis and interpretation of data. $\mathrm{KG}, \mathrm{HO}$ and NN contributed to acquisition of data. KK made substantial contributions to conception and design. MI contributed to the analysis and interpretation of data. All authors read and approved the final manuscript.

\section{Ethics approval and consent to participate}

The present study was approved by the Animal Care Committee of Juntendo University (Tokyo, Japan; registration no. 1398; approval no. 310212).

\section{Patient consent for publication}

Not applicable. 


\section{Competing interests}

The authors declare that they have no competing interests.

\section{References}

1. Iijima Y, Ajiki T, Murayama A and Takeshita K: Effect of artificial nerve conduit vascularization on peripheral nerve in a necrotic bed. Plast Reconstr Surg Glob Open 4: e665, 2016.

2. Kornfeld T, Vogt PM and Radtke C: Nerve grafting for peripheral nerve injuries with extended defect sizes. Wien Med Wochenschr 169: 240-251, 2019.

3. Rbia N, Bulstra LF, Saffari TM, Hovius SER and Shin AY: Collagen nerve conduits and processed nerve allografts for the reconstruction of digital nerve gaps: A single-institution case series and review of the literature. World Neurosurg 127: e1176-e1184, 2019.

4. Siemionow M, Cwykiel J, Uygur S, Kwiecien G, Oztürk C Szopinski J and Madajka M: Application of epineural sheath conduit for restoration of $6-\mathrm{cm}$ long nerve defects in a sheep median nerve model. Microsurgery 39: 332-339, 2018.

5. Biasibetti E, Bisanzio D, Mioletti S, Amedeo S, Iuliano A, Bianco P and Capucchio MT: Spontaneous age-related changes of peripheral nerves in cattle: Morphological and biochemical studies. Anat Histol Embryol 45: 100-108, 2015.

6. Goto K, Naito K, Nakamura S, Nagura N, Sugiyama Y, Obata H, Kaneko A and Kaneko K: Protective mechanism against age-associated changes in the peripheral nerves. Life Sci 253 $117744,2020$.

7. Novak CB, Anastakis DJ, Beaton DE, Mackinnon SE and Katz J: Biomedical and psychosocial factors associated with disability after peripheral nerve injury. J Bone Joint Surg Am 93: 929-936, 2011.

8. Fields RD, Le Beau JM, Longo FM and Ellisman MH: Nerve regeneration through artificial tubular implants. Prog Neurobiol 33: 87-134, 1989.

9. Painter MW, Lutz AB, Cheng YC, Latremoliere A, Duong K, Miller CM, Posada S, Cobos EJ, Zhang AX, Wagers AJ, et al: Diminished schwann cell repair responses underlie age-associated impaired axonal regeneration. Neuron 83: 331-343, 2014.

10. Verdú E, Ceballos D, Vilches JJ and Navarro X: Influence of aging on peripheral nerve function and regeneration. J Peripehr Nerv Syst 5: 191-208, 2000.

11. Pola R, Aprahamian TR, Bosch-Marcé M, Curry C, Gaetani E, Flex A, Smith RC, Isner JM and Losordo DW: Age-dependent VEGF expression and intraneural neovascularization during regeneration of peripheral nerves. Neurobiol Aging 25: 1361-1368, 2004.
12. Cattin AL, Burden JJ, Van Emmenis L, Mackenzie FE, Hoving JJ, Garcia Calavia N, Guo Y, McLaughlin M, Rosenberg LH, Quereda V, et al: Macrophage-induced blood vessels guide Schwann cell-mediated regeneration of peripheral nerves. Cell 162: 1127-1139, 2015.

13. Nishida Y, Yamada Y, Kanemaru H, Ohazama A, Maeda T and Seo K: Vascularization via activation of VEGF-VEGFR signaling is essential for peripheral nerve regeneration. Biomed Res 39: 287-294, 2018.

14. Fujiwara S, Hoshikawa S, Ueno T, Hirata M, Saito T, Ikeda T, Kawaguchi H, Nakamura K, Tanaka S and Ogata T: SOX10 transactivates S100B to suppress Schwann cell proliferation and to promote myelination. PLoS One 9: e115400, 2014.

15. Jessen KR and Mirsky R: Signals that determine Schwann cell identity. J Anat 200: 367-376, 2002.

16. Kuhlbrodt K, Herbarth B, Sock E, Hermans-Borgmeyer I and Wegner M: Sox 10, a novel transcriptional modulator in glial cells. J Neurosci 18: 237-250, 1998.

17. Budni J, Bellettini-Santos T, Mina F, Garcez ML and Zugno AI: The involvement of BDNF, NGF and GDNF in aging and Alzheimer's disease. Aging Dis 6: 331-341, 2015.

18. Chen ZW and Wang MS: Effects of nerve growth factor on crushed sciatic nerve regeneration in rats. Microsurgery 16: 547-551, 1995.

19. Hasenöhrl RU, Söderstróm S, Mohammed AH, Ebendal T and Huston JP: Reciprocal changes in expression of mRNA for nerve growth factor and its receptors TrkA and LNGFR in brain of aged rats in relation to maze learning deficits. Exp Brain Res 114: 205-213, 1997.

20. Kemp SW, Webb AA, Dhaliwal S, Syed S, Walsh SK and Midha R: Dose and duration of nerve growth factor (NGF) administration determine the extent of behavioral recovery following peripheral nerve injury in the rat. Exp Neurol 229: 460-470, 2011.

21. Lärkfors L, Ebendal T, Whittemore SR, Persson H, Hoffer B and Olson L: Decreased level of nerve growth factor (NGF) and its messenger RNA in the aged rat brain. Brain Res 427: 55-60, 1987.

22. Grinsell D and Keating CP: Peripheral nerve reconstruction after injury: A review of clinical and experimental therapies. Biomed Res Int 2014: 698256, 2014.

23. Hall S: Nerve repair: A neurobiologist's view. J Hand Surg Br 26: 129-136, 2001

24. Mokuno K, Sobue G, Reddy UR, Wurzer J, Kreider B, Hotta H, Baron P, Ross AH and Pleasure D: Regulation of Schwann cell nerve growth factor receptor by cyclic adenosine 3',5'-monophosphate. J Neurosci Res 21: 465-472, 1988.

This work is licensed under a Creative Commons

Attribution-NonCommercial-NoDerivatives 4.0 International (CC BY-NC-ND 4.0) License. 